\title{
Impact of Low Maternal Education on Early Childhood Overweight and Obesity in Europe
}

\author{
Milagros Ruiz, ${ }^{\text {a }}$ Peter Goldblatt, ${ }^{\mathrm{b}}$ Joana Morrison, ${ }^{\mathrm{a}}$ Daniela Porta, ${ }^{\mathrm{c}}$ Francesco Forastiere, ${ }^{\mathrm{c}}$ Daniel Hryhorczuk, ${ }_{1}^{\mathrm{d}}$ Youriy Antipkin, ${ }^{\mathrm{e}}$ \\ Marie-Josèphe Saurel-Cubizolles, ${ }^{\mathrm{f}}$ Sandrine Lioret, ${ }^{\mathrm{g}}$ Martine Vrijheid, ${ }^{\mathrm{h}, \mathrm{i}}$ Maties Torrent, ${ }^{\mathrm{i}, \mathrm{j}}$ Carmen Iñiguez, $^{\mathrm{k}}$ Isabel Larrañaga, ${ }^{\mathrm{l}, \mathrm{m}}$ \\ Chryssa Bakoula, ${ }^{\mathrm{n}}$ Alexandra Veltsista, ${ }^{\mathrm{n}}$ Manon van Eijsden, ${ }^{\circ}$ Tanja G. M. Vrijkotte, ${ }^{\mathrm{p}}$ Lenka Andrýsková, ${ }^{\mathrm{q}}$ Ladislav Dušek, ${ }^{\mathrm{q}, \mathrm{r}}$ \\ Henrique Barros, ${ }^{\mathrm{s}, \mathrm{t}}$ Sofia Correia, ${ }^{\mathrm{s}, \mathrm{t}}$ Marjo-Riitta Järvelin, ${ }_{1}^{\mathrm{u}, \mathrm{v}, \mathrm{w}, \mathrm{x}}$ Anja Taanila, ${ }^{\mathrm{y}}$ Johnny Ludvigsson, ${ }^{\mathrm{z}}$ Tomas Faresjö, ${ }^{\text {aa }}$ \\ Michael Marmot, ${ }^{a, b}$ Hynek Pikhart ${ }^{a}$ \\ ${ }^{a}$ Research Department of Epidemiology and Public Health, \\ ${ }^{b}$ Research Department of Epidemiology and Public Health, UCL Institute of Health Equity, University College London, London, UK \\ ${ }^{c}$ Department of Epidemiology, Lazio Regional Health System, Rome, Italy \\ ${ }^{d}$ Center for Global Health, University of Illinois College of Medicine, Chicago, IL, USA \\ ${ }^{e}$ Institute of Pediatrics, Obstetrics, and Gynecology, Kyiv, Ukraine \\ ${ }^{f}$ Institut National de la Santé et de la Recherche Médicale (INSERM) UMR 1153, Obstetrical, Perinatal and Pediatric Epidemiology Research Team \\ (Epopé), Center for Epidemiology and Statistics, Sorbonne Paris Cité, DHU Risks in Pregnancy, \\ ${ }^{8}$ Institut National de la Santé et de la Recherche Médicale (INSERM) UMR 1153, Early Origin of the Child's Health and Development Team \\ (ORCHAD), Center for Epidemiology and Statistics, Sorbonne Paris Cité, Paris Descartes University, Paris, France \\ ${ }^{h}$ Center for Research in Environmental Epidemiology (CREAL), \\ ${ }^{i}$ Spanish Consortium for Research on Epidemiology and Public Health (CIBERESP), Barcelona, \\ ${ }^{j} I B-S a l u t$ Menorca Health Area, Balearic Islands, \\ ${ }^{k}$ FISABIO - Universitat Jaume I - Universitat de València Joint Research Unit of Epidemiology and Environmental Health, Castellón de la Plana, \\ ${ }^{l}$ Public Health Department of Gipuzkoa, Gipuzkoa, \\ ${ }^{m}$ BIODONOSTIA Health Research Institute, San Sebastian, Spain \\ ${ }^{n}$ First Department of Paediatrics, Aghia Sophia Children's Hospital, University of Athens, Athens, Greece \\ ${ }^{\circ}$ Department of Epidemiology and Health Promotion, Public Health Service of Amsterdam, \\ ${ }^{p}$ Department of Public Health, Academic Medical Center, University of Amsterdam, Amsterdam, The Netherlands \\ ${ }^{q}$ Faculty of Science, Research Centre of Toxic Compounds in the Environment (RECETOX), \\ ${ }^{r}$ Institute of Biostatistics \& Analyses (IBA), Masaryk University, Brno, Czech Republic \\ ${ }^{s}$ EPIUnit - Institute of Public Health, University of Porto, \\ ${ }^{t}$ Department of Clinical Epidemiology, Predictive Medicine and Public Health, University of Porto Medical School, Porto, Portugal \\ ${ }^{u}$ Department of Epidemiology and Biostatistics, MRC Health Protection Agency (HPE), Centre for Environment and Health, School of Public \\ Health, Imperial College London, London, UK \\ ${ }^{v}$ Biocenter Oulu, University of Oulu, \\ ${ }^{w}$ Unit of Primary Care, Oulu University Hospital, \\ ${ }^{x}$ Faculty of Medicine, Center for Life Course Epidemiology, \\ Institute of Health Sciences, University of Oulu, Oulu, Finland \\ ${ }^{z}$ Division of Pediatrics, Department of Clinical and Experimental Medicine, \\ ${ }^{a a}$ Department of Medicine and Health, Community Medicine/General Practice Faculty of Health Sciences, Linköping University, Linköping, Sweden
}

\begin{abstract}
Background: Comparable evidence on adiposity inequalities in early life is lacking across a range of European countries. This study investigates whether low maternal education is associated with overweight and obesity risk in children from distinct European settings during early childhood.

Methods: Prospective data of 45413 children from 11 European cohorts were used. Children's height and weight obtained at ages 4-7 years were used to assess prevalent overweight and obesity according to the International Obesity Task Force definition. The Relative/Slope Indices of Inequality (RII/SII) were estimated within each cohort and by gender to investigate adiposity risk among children born to mothers with low education as compared to counterparts born to mothers with high education. Individual-data meta-analyses were conducted to obtain aggregate estimates and to assess heterogeneity between cohorts.
\end{abstract}


Results: Low maternal education yielded a substantial risk of early childhood adiposity across 11 European countries. Low maternal education yielded a mean risk ratio of 1.58 (95\% confidence interval (CI) $1.34,1.85)$ and a mean risk difference of $7.78 \%(5.34,10.22)$ in early childhood overweight, respectively, measured by the RII and SII. Early childhood obesity risk by low maternal education was as substantial for all cohorts combined (RII = 2.61 $(2.10,3.23))$ and $(\mathrm{SII}=4.01 \%(3.14,4.88))$. Inequalities in early childhood adiposity were consistent among boys, but varied among girls in a few cohorts.

Conclusions: Considerable inequalities in overweight and obesity are evident among European children in early life. Tackling early childhood adiposity is necessary to promote children's immediate health and well-being and throughout the life course.

Keywords: child, preschool, child development, cohort studies, comparative study, Czech Republic, epidemiology, Europe, Finland, France, Greece, health inequalities, Italy, maternal educational status, meta-analysis, Netherlands, obesity, overweight, Portugal, Spain, Sweden, United Kingdom, Ukraine.

Early childhood adiposity generally tracks through the life course and is linked with poor metabolic risk profiles and chronic diseases in later life. ${ }^{1-3}$ While social inequalities in overweight and obesity risk have been well-established among school-age and adolescent European children, there is limited comparable evidence available for younger (3-7 years) children during early child hood across Europe. ${ }^{4,5}$

The complex determinants of early childhood overweight and obesity are depicted by the Total Environment Assessment Model of Early Childhood. ${ }^{6}$ Social and environmental factors at the individual-, family-, residential-, and broader national and regional levels influence overweight and obesity risk in both an independent and intertwined manner. Established risk factors across these levels include: poor maternal health and particularly maternal obesity; unhealthy behaviours during pregnancy and during childrearing; impaired foetal development, feeding practices at infancy and related diet; sedentary and sleep behaviour; alongside neighbourhood conditions such as safety and access to green spaces; to the availability, cost, and formulation of food choices; and the marketing environment. ${ }^{7-9}$ Extensive research has documented how these factors lead to inequalities in early childhood overweight and obesity in highincome countries, including those in Europe. ${ }^{5,7,10,11}$ While there is recognition that these established risk factors are socially patterned, it is unknown whether

\section{Correspondence:}

Milagros Ruiz, Research Department of Epidemiology and Public Health, University College London, 1-19 Torrington Place, London WC1E 6BT, UK.

E-mail: milagros.ruiz.10@ucl.ac.uk boys and girls differ in their susceptibility to certain social and environmental conditions.

Greater educational attainment among mothers may curtail the child's vulnerability to the cumulative nature of these obesogenic factors, through greater use of positive parenting practices and enhanced acquisition of the familial and residential environment, and a reduced likelihood of other disadvantages, such as belonging to a lone parent family and living in a deprived household or community. ${ }^{7,12}$ Low maternal education has been closely linked to well-established life course predictors of early childhood adiposity, including smoking during pregnancy, foetal growth restriction, limited breast feeding, and food insecurity; as well as psycho-social stressors related to familial functioning and material insecurity. 5,10,12,13

High maternal education has been linked with a reduced likelihood of adiposity among children during early childhood in some European populations, according to a recent systematic review of inequalities in early childhood health and development in Europe. ${ }^{5}$ As current evidence is primarily limited to individual-country studies and is disproportionately based on Western and Northern European populations, it is difficult to ascertain whether associations are consistent during the early childhood period across countries with unique obesogenic landscapes. Moreover, the potential role of gender upon the effect of established risk factors on early childhood adiposity has been overlooked in the literature. ${ }^{14}$ The objective of this study is to undertake a comparative examination of the association between maternal education and subsequent risk of both overweight and obesity among children aged 4-7 in 11 European 
birth cohorts, and to assess potential effect modification by gender.

\section{Methods}

\section{Data sources}

The DRIVERS for Health Equity research programme included a European cross-cohort study of inequalities in early child health and development. The present analysis draws on this component of DRIVERS, and is based on 11 European birth cohorts (in ascending order of child's age at measurement): the Gene and Environment Prospective Study on Infancy in Italy, Italy (IT-GASPII, $N=543$ ); the Environment and Childhood Project, Spain (ES-INMA, $N=1726$ ); the Generation XXI Study, Portugal (PT-G21, $N=5686$ ); the Millennium Cohort Study, United Kingdom (UK-MCS, $N=14$ 186); the All Babies in Southeast Sweden Study (SE-ABIS, $N=6494$ ); the motherchild Study of Pre- and Post-natal Determinants of Child Growth, Development, and Health, France (FREDEN, $N=1139$ ); the Amsterdam Born Children and their Development Study, Netherlands (NL-ABCD, $N=3245$ ); the European Longitudinal Study of Pregnancy and Childhood, Czech Republic (CZ-ELSPAC, $N=3665$ ); the Family and Children of Ukraine Study, Ukraine (UA-FCOU, $N=925$ ); the Greek Birth Cohort, Greece (GR-GBC, $N=1843$ ); and the Northern Finland Birth Cohort 1985/1986 Study, Finland (FI-NFBC8586, $N=5961$ ). Details of participating cohorts including eligibility criteria have been previously reported. ${ }^{15}$ Participants consisted of 45413 children born between April 1983 and October 2006, and for whom comparable data of maternal education, height and weight during early childhood were available.

\section{Children's and maternal variables}

Height and weight were obtained when children were aged between 4 and 7 years and measured by trained personnel in all cohorts, with the exception of GR-GBC and SE-ABIS. Data on these two items in both cohorts were based on parental reports, which were found to be comparable with measured height and weight in sub-sample studies. ${ }^{16,17}$ Body mass index (BMI) was calculated by dividing the child's weight in kilograms by the square of their height $(\mathrm{kg} /$ $\mathrm{m}^{2}$ ). Overweight cases were defined using the ageand gender-specific thresholds for BMI recommended by the International Obesity Task Force (IOTF) in each cohort, which correspond to a BMI value of $25 \mathrm{~kg} / \mathrm{m}^{2}$ at 18 years of age. Supporting analyses on obese cases in each cohort were also defined by the IOTF as the age- and gender-specific BMI values that are comparable to an adult BMI value of $30 \mathrm{~kg} / \mathrm{m}^{2} .{ }^{18}$

Mother's education was ascertained at entry to each cohort study, either during pregnancy or near the time of birth. Years of schooling were available in FREDEN, GR-GBC, NL-ABCD, and PT-G21. Levels of completed schooling were collected in IT-GASPII, UA-FCOU, ES-INMA, CZ-ELSPAC, FI-NFBC8586, and SE-ABIS. Highest obtained qualifications were obtained in UK-MCS. The International Standard Classification of Education (ISCED) is an international categorisation managed by the United Nations Educational, Scientific, and Cultural Organization to facilitate comparison of educational data across countries. The country-specific coding scheme provided by ISCED-1997 was used to classify mothers into the following categories: (i) post-secondary non-tertiary to second stage of tertiary education (ISCED 4-6), (ii) upper secondary education (ISCED 3), and (iii) preprimary to lower secondary or second stage of basic education (ISCED 0-2). ${ }^{19}$

Age, gender, and maternal age at study baseline were available in all participating cohorts and were included in the present analysis. Due to differences in the inclusion criteria between cohorts, foreign-born mothers or those belonging to an ethnic minority group were present in 7 of the 11 analytic samples. Data of mother's country of birth were available in IT-GASPII, FR-EDEN, ES-INMA, and NL-ABCD. PT-G21 assessed mother's country of birth and migration history of mothers and her parents. In UK-MCS, perceived ethnicity was ascertained. These data were dichotomised and used as a proxy of mother's foreign-born or ethnic minority status.

\section{Statistical analyses}

Analyses were carried out among singleton participants, and study characteristics were assessed in each cohort (Table 1). The cohort-specific distribution of mother's education was directly age-standardised using the WHO European Standard Population to account for variation in maternal age across samples. ${ }^{20}$ Early childhood overweight and obesity prevalence by mother's education was calculated for each cohort. The $X^{2}$ test for trend assessed linearity 


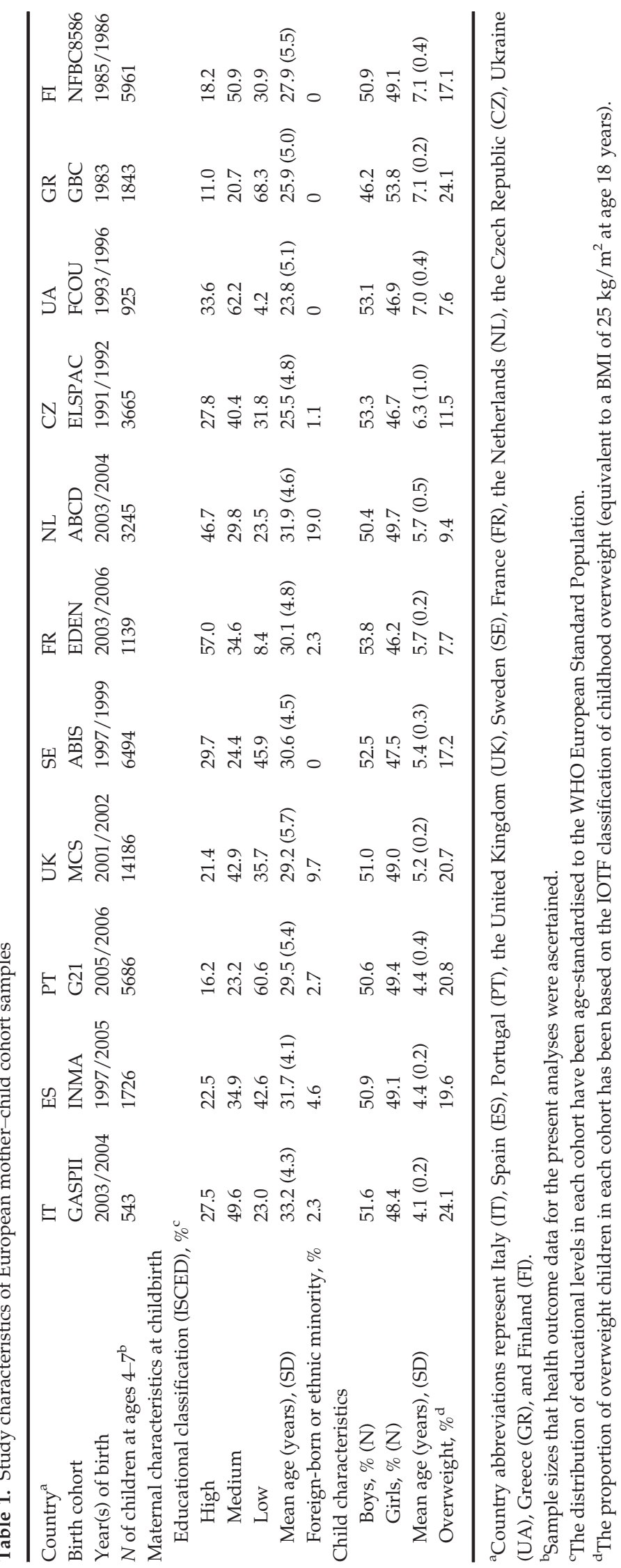


across educational groups, and separately for boys and girls (Tables 2 and S1).

Associations between maternal education and early childhood overweight and obesity were estimated to infer relative and absolute social inequalities in each cohort sample, by using the Relative (and Slope) Indices of Inequality (RII/SII) respectively. These regression-based indices take into account both the size and distribution of social groups across the population. ${ }^{21}$ The RII is a summary measure of relative inequality, defined as the risk ratio of the outcome between children at the lowest and those at the highest end of the maternal education hierarchy. The SII is the corresponding measure of absolute inequality, defined as the risk difference in prevalence of the child outcome between the two ends of this hierarchy. A RII score greater than 1 (and a SII score greater than 0) indicates the presence of inequality between low and high positions. For both indices, higher scores denote a larger magnitude of inequality. Given the extent of cohort differences in maternal education, these indices account for such differences as a source of variation in the magnitude of health inequalities and facilitate comparison of estimates between cohorts.

Generalised linear models were performed to obtain the RII and SII by, respectively, specifying a logarithmic or an identity link function. Educational categories were ranked from high to low and were each assigned a value between 0 and 1 , based on the cumulative percentage of the mid-point of the ranges observed for each cohort. Firstly, models were adjusted for age, gender, maternal age, and ethnicity, as appropriate, for each outcome across cohorts. Sensitivity testing of these results were compared to associations with BMI $\left(\mathrm{kg} / \mathrm{m}^{2}\right)$ and adjusted for the same co-variates. Secondly, interaction terms between maternal education and gender were added to the models to assess effect modification by gender. Thirdly, as gender moderated the effect estimates among a few cohorts, models were then stratified by gender. Cohort- and gender-specific scores were pooled together to obtain mean RII and SII scores for boys and girls at the aggregate level using random-effects meta-analysis procedures (Figures 1 and S1). The degree of heterogeneity was tested using the $I^{2}$ statistic and confirmed the a priori expectation of heterogeneity between cohorts that is not attributable to sampling variation. Meta-analysis findings were subsequently studied by random-effects meta-regression, in order to further assess effects moderated by gender.

Descriptive and regression analyses of the UK-MCS employed survey weights to account for the cohort's sampling design. ${ }^{22}$ Regression analyses of ES-INMA included geographic area as an additional co-variate to incorporate the multicentre design of the cohort. All analyses were carried out using STATA version 13 (Stata Corp, College Station, TX, USA).

\section{Ethics}

Study protocols were approved by ethics committees for each cohort. All participating mothers provided informed consent for themselves and their children. Maternal characteristics obtained at study baseline

Table 2. Prevalence of early childhood overweight by mother's educational level in 11 European birth cohorts, by gender

\begin{tabular}{|c|c|c|c|c|c|c|c|c|c|c|c|}
\hline Country $^{a}$ & IT & ES & PT & UK & SE & FR & NL & $\mathrm{CZ}$ & UA & GR & FI \\
\hline Birth cohort & GASPII & INMA & G21 & MCS & ABIS & EDEN & $\mathrm{ABCD}$ & ELSPAC & FCOU & GBC & NFBC8586 \\
\hline \multicolumn{12}{|l|}{ Overweight, \% } \\
\hline \multicolumn{12}{|l|}{ Boys } \\
\hline High & 13.7 & 14.6 & 10.7 & 16.3 & 11.9 & 4.2 & 4.5 & 8.1 & 7.1 & 22.1 & 12.7 \\
\hline Medium & 18.8 & 19.1 & 15.5 & 19.6 & 15.3 & 8.1 & 10.2 & 11.4 & 5.8 & 19.6 & 15.7 \\
\hline Low & 29.4 & 17.5 & 18.4 & 19.3 & 18.2 & 3.8 & 21.2 & 11.9 & 11.1 & 22.5 & 16.6 \\
\hline$P$ for trend ${ }^{b}$ & 0.047 & 0.414 & $<0.001$ & 0.017 & $<0.001$ & 0.160 & $<0.001$ & 0.047 & 0.761 & 0.597 & 0.041 \\
\hline \multicolumn{12}{|l|}{ Girls } \\
\hline High & 37.8 & 22.3 & 17.4 & 20.0 & 18.4 & 7.2 & 7.2 & 9.6 & 16.7 & 25.2 & 16.0 \\
\hline Medium & 24.2 & 22.6 & 27.8 & 23.7 & 18.8 & 13.5 & 16.5 & 11.2 & 8.1 & 27.1 & 19.2 \\
\hline Low & 33.3 & 21.1 & 30.5 & 26.1 & 21.5 & 21.7 & 25.3 & 15.2 & 3.7 & 26.1 & 21.5 \\
\hline$P$ for trend ${ }^{b}$ & 0.208 & 0.755 & $<0.001$ & $<0.001$ & 0.075 & 0.003 & $<0.001$ & 0.005 & $0.022 \uparrow$ & 0.980 & 0.008 \\
\hline
\end{tabular}

${ }^{a}$ Country abbreviations represent Italy (IT), Spain (ES), Portugal (PT), the United Kingdom (UK), Sweden (SE), France (FR), the Netherlands (NL), the Czech Republic (CZ), Ukraine (UA), Greece (GR), and Finland (FI).

${ }^{\mathrm{b}} P$-values below or equal to 0.05 are embolded. $\uparrow$ Denotes a positive association between maternal education and risk of overweight. 


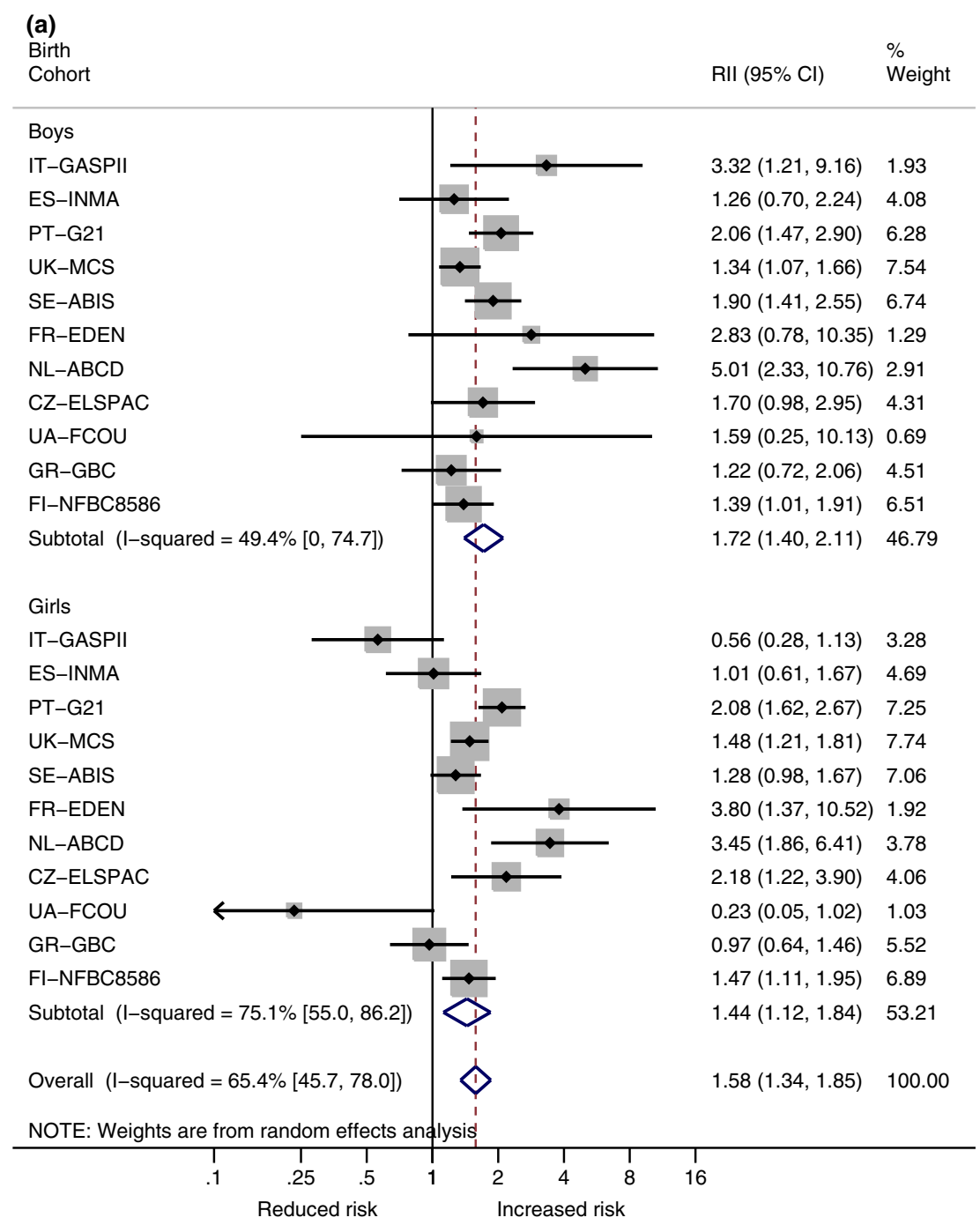

Figure 1. (a) Forest plot of combined RII analyses of relative inequalities in early childhood overweight risk in Europe, by gender; a score greater than 1 (RII, risk ratio) indicates inequality between low and high positions of maternal education.

and child height and weight acquired between the ages of 4-7 were provided from each cohort in accordance with the ethical procedures approved for each site.

\section{Results}

Mean mother's age ranged between 23.8 and 33.2 years at the time of childbirth (Table 1). Due to differences in study design, the proportion of foreignborn mothers was nil in four analytic samples, and elsewhere ranged from 1.1\% in CZ-ELSPAC to $19 \%$ in NL-ABCD. Considerable proportions of mothers with low education were present in GR-GBC (68.3\%), PT-G21 (60.6\%), and ES-INMA (42.6\%). Mothers with high education comprised a third or more of samples in SE-ABIS, FR-EDEN, NL-ABCD, and UA-FCOU. The prevalence of overweight children was lowest in UA-FCOU (7.6\%) and highest in GR-GBC and IT-GASPII (24.1\%).

Table 2 reports the prevalence distribution of overweight by mother's educational level by cohort and gender. Early childhood gradients in prevalent overweight among boys were statistically significant in IT-GASPII, PT-G21，UK-MCS， SE-ABIS，NL-ABCD, CZ-ELSPAC, and FI-NFBC8586. Among girls, gradients in early childhood overweight were significant in PT-G21, UK-MCS, FR-EDEN, NL-ABCD, CZ-ELSPAC, UA-FCOU, and FI-NFBC8586. In the case of UA-FCOU, the gradient indicated a higher risk of 


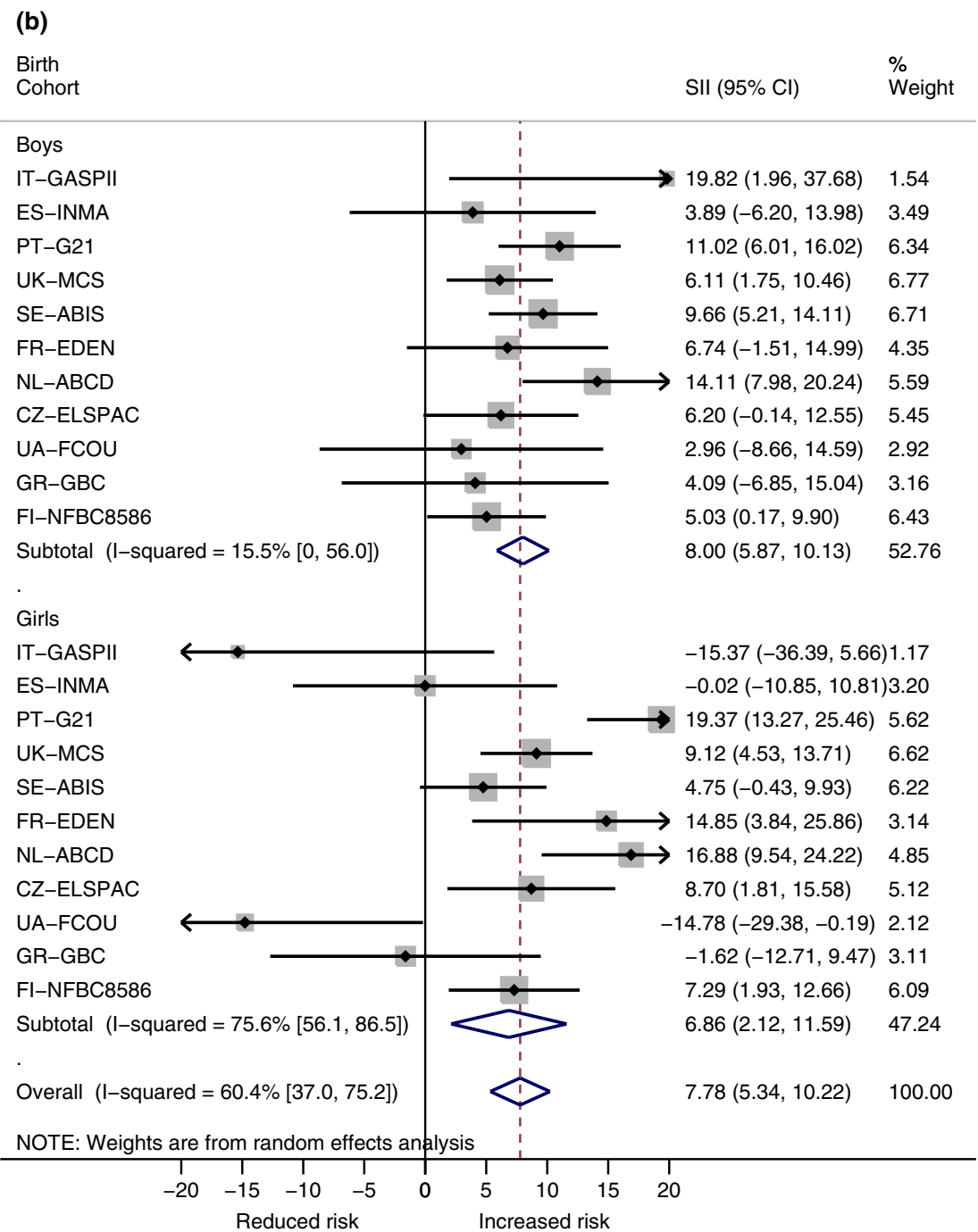

Figure 1. (b) Forest plot of combined SII analyses of absolute inequalities in early childhood overweight risk in Europe, by gender; a score greater than 0 (SII, risk difference) indicates inequality between low and high positions of maternal education.

overweight among girls with mothers with higher levels of education. Elsewhere, overweight gradients were not detected by the $X^{2}$ test for trend, which may be due to limited study power among some cohorts.

Associations between mother's education and the prevalence of early childhood overweight were assessed within each cohort and adjusted for age and mother's age and ethnicity (as appropriate). Significant effect modification by gender was observed in relative (RII) and absolute (SII) inequalities among a few cohorts. As compared to boys, SII estimates were notably higher among girls in PT-G21 for overweight ( $P=0.033$, test for interaction). SII estimates for overweight status also indicated gender differences in IT-GASPII $(P=0.016$, test for interaction) and UA-FCOU ( $P=0.045$, test for interaction). However, in these two cases, the SIIs indicated a higher risk of overweight among girls with mothers with higher level of education. Gender differences were also found for relative (RII) inequalities in early childhood overweight in IT-GASPII $(P=0.016$, test for interaction) and SE-ABIS ( $P=0.048$, test for interaction).

Sensitivity analyses using BMI $\left(\mathrm{kg} / \mathrm{m}^{2}\right)$ as a continuous outcome generated similar results to those of overweight prevalence in each cohort (available upon request) and addressed the concern of Type II error among smaller samples or those with fewer cases.

Figure 1 displays the combined RII and SII analyses across cohorts and by gender. Overall, children of low-educated mothers were 1.58 times (RII) more likely to be overweight than counterparts of mothers 
with high education, and corresponds to a mean risk difference of $7.8 \%$ (SII). Among boys, RIIs ranged from 1.22 in GR-GBC to 5.01 in NL-ABCD, combining to a mean risk ratio of 1.72 . SIIs varied between $3.0 \%$ in UA-FCOU and $19.8 \%$ in IT-GASPII, and resulted in an average risk difference of $8.0 \%$. Between cohort heterogeneity $\left(I^{2}\right)$ was modest among boys, as shown by the proportion and $95 \%$ confidence interval estimates of $49.4 \%$ (95\% CI: 0, 74.7) and 15.5\% (95\% CI: 0, 56.0), respectively, for the RII/SII. Among girls, positive associations between low maternal education and lower risk of overweight were shown in IT-GASPII, UA-FCOU, and GR-GBC. RIIs of overweight among girls extended from 0.23 in UA-FCOU to 3.8 in FREDEN, which aggregated to an average risk ratio of 1.44. Absolute inequalities (SIIs) in prevalent overweight spanned from a risk difference of $-15.4 \%$ among girls in IT-GASPII to $19.4 \%$ among girls in PTG21, which pooled to a mean risk difference of $6.86 \%$ across cohorts. Between cohort differences $\left(I^{2}\right)$ were appreciable among girls, as denoted by the per cent and interval estimates for the mean RII/SII: $65.4 \%$ (95\% CI: $45.7,78.0)$ and 75.6\% (95\% CI: 56.1, 86.5).

Despite these differences in the combined overweight analysis, meta-regression found no substantial source of between cohort heterogeneity that was attributable to gender ( $P=0.21$ for RII; $P=0.90$ for SII).

Supporting obesity analyses showed that the prevalence ranged from $1.1 \%$ in FR-EDEN to $6.5 \%$ in PT-G21. Gradients in obesity risk among boys and girls in each cohort were generally similar to those for overweight status (Table S1). On average, children of mothers with low education were 2.61 times (RII) more likely to be obese than those born to mothers with high education, which relates to an average risk difference in obesity of $4.0 \%$ in absolute terms (SII) (Figure S1). Among girls, RIIs culminated to a 2.42 greater mean likelihood of obesity, and SIIs combined to mean risk difference of $3.98 \%$ for all cohorts combined. Statistical evidence of between cohort heterogeneity $\left(I^{2}\right)$ in relative (RII) and absolute (SII) obesity inequalities was not found among boys or girls.

\section{Discussion}

To our knowledge, this is the first comparative study to examine the association between mother's education and early childhood adiposity among a multitude of birth cohorts, and to prominently demonstrate these inequalities in the European region. Substantial inequalities in both overweight and obesity risk were observed at the aggregate level. Non-significant findings observed among some cohorts may be related to factors of statistical power, such as analytic sample sizes, prevalence rates of early childhood overweight/obesity, and the true magnitude of associations.

Previous regional work by the IDEFICS Consortium used cross-sectional surveys to assess adiposity risk by maternal education among children aged $4-5$ in Belgium, Cyprus and Italy, and found that inequalities varied by gender in a few cases. ${ }^{23}$ In parallel with these and other individual-level findings, the present study has demonstrated some inconsistency by gender in overweight inequalities among several cohorts. ${ }^{23-25}$ The social patterning of early childhood adiposity risk factors has been shown to differ throughout Europe. ${ }^{26}$ For example, children of mothers with higher levels of education in Ukraine have shown to be more likely to consume greater quantities of expensive foods, such as red and processed meats, and thus exceed recommended levels of protein and cholesterol. ${ }^{25}$ Increased protein intake has been shown to predict increased adiposity among children in early childhood, and the endocrine response to a high-protein diet appears to be stronger among girls. ${ }^{27,28}$ Physical inactivity has also been linked to overweight and obesity during early childhood, and increased sedentary behaviour among young girls is wellestablished. Cultural norms and gendered expectations of physical activity during the early years may explain the extent to which inequalities differ across participating cohorts. ${ }^{29,30}$

Obesogenic factors vary by broader environmental conditions that promote high energy intake and sedentary behaviour. These health behaviours are widely influenced by the built landscape, economic and political determinants and sociocultural traditions. ${ }^{31}$ Taking the Ukrainian findings as example, obesity has been linked to socio-economic privilege preceding the fall of communism and to susceptibility to the free-market economy in Eastern Europe. ${ }^{32}$ Elsewhere, the general consistency of findings across cohorts from unique obesogenic environments and at different stages of the nutrition transition is noteworthy. As early childhood adiposity inequalities were demonstrated among children born over a 20-year period, children and their mothers were subject to different environmental conditions that may have influenced the magnitude of inequalities by 
maternal education. Despite the observed differences in the RII and SII estimates between cohorts, the meta-analyses showed that estimated inequalities were not significantly heterogeneous for obese children, which underscores the impermeable risk attributed to low maternal education in early life. Estimated inequalities in early childhood overweight were shown to significantly differ between cohorts only among girls, which suggest some modification due to changes in obesogenic factors and broader conditions.

Pre-eminent inequalities in the Netherlands may relate to the large proportion of foreign-born mothers, which was linked with a higher risk of early childhood adiposity in this cohort. ${ }^{33}$ Bias may occur as the cohorts required participation from birth to early childhood age, where follow-up rates were lower in the Netherlands and Ukraine. Missing height and weight data were greater for children of mother's with low education in the Czech Republic, Greece, and Portugal; which may underestimate inequalities in these cohorts. Parsimonious adjustment by child age, maternal age, and foreign-born/ethnic minority status, does not eliminate residual confounding by factors unavailable for this analysis, such as markers of maternal health and other characteristics. For example, inequalities in early childhood adiposity by mother's education may be explained by maternal obesity, as rates tend to be higher among mothers with less education. Although data on mother's BMI prior to pregnancy were not available in all analytic cohort samples, this was accessible in the NL-ABCD and UK-MCS for the present study. RII and SII estimates were marginally attenuated and remained statistically significant after inclusion of maternal BMI in these cohorts. Analysis of children born over a 20year period may pose a methodological limitation, but these cohorts are among the most viable in the region, including some of the first to be established in their respective countries. Comparable findings of early childhood overweight and obesity using the same case definitions and methods of analyses on eleven European birth cohorts overcame the acknowledged gap in this area of evidence for the region, due to insufficient data sources and contrasting definitions employed between countries that make surveillance at the regional level problematic. Assessment by birth year of the children did not indicate a particular trend of inequalities in early childhood overweight and obesity.
This comparative study has established a greater risk of early childhood overweight and obesity by mother's education at the European level. Children's risk of adiposity firmly illustrates the strength of the social environment, and the limited reach of traditional prevention strategies. ${ }^{26}$ The extent to which these inequalities manifest in children's early life has important policy implications. Firstly, the educational attainment of women and girls is a crucial social determinant for the health and well-being of offspring, given greater socio-economic resources, and enhanced facilities among mothers with higher levels of education. Our findings suggest that progress towards the Europe 2020 education targets to curtail the rates of early school leaving and to expand the share of young and older adults with tertiary education may have a beneficial impact on children's health of future mothers. ${ }^{34}$ Actions to reduce overweight and obesity inequalities in women of childbearing age may work to reduce the inter-generational transmission of obesity risk from mothers to children. Secondly, in addition to school-based interventions, coordinated efforts need to be tailored to the family, and the home environment to minimise the social gradient in overweight and obesity present well before children enter compulsory education. ${ }^{35}$ Interventions targeted to mothers with lower levels of education may be implemented during pregnancy and following childbirth to offset risk factors of early childhood adiposity. For example, efforts aimed to lower prenatal growth restriction and rapid growth acceleration during infancy may improve the trajectory of adiposity throughout the life course, as well as those that promote prolonged and exclusive breast feeding, and timely and complementary feeding may also have a substantial effect on weight gain and body fatness during early childhood.

Overweight and obesity risk among European children during early childhood was greater among those born to mothers with low education. Social care and public health efforts must be accelerated to reduce these inequalities and to curtail further gaps through the life course.

\section{Author contributions}

MR designed the study, carried out all analyses, wrote the initial manuscript, and approved the final manuscript as submitted. PG critically reviewed the study, provided important theoretical contributions to the 
initial manuscript, and approved the final manuscript as submitted. JM and MM critically reviewed and revised the manuscript, and approved the final manuscript as submitted. DP, FF, DH, YA, M-JS-C, SL, MV, MT, CI, IL, CB, AV, ME, TGMV, LA, LD, HB, SC, $\mathrm{M}-\mathrm{RJ}, \mathrm{AT}, \mathrm{JL}$, and TF procured and coordinated data for this study, critically reviewed the manuscript, made important contributions to its intellectual content, and approved the final manuscript as submitted. HP conceptualised and designed the study, oversaw all stages of the analyses, guided the initial manuscript, and approved the final manuscript as submitted.

\section{Acknowledgements}

We thank the participating members of the European birth cohort studies included in the present analysis. We would like to acknowledge Marie-Aline Charles, Anne Forhan, and Barbara Heude from FR-EDEN. We also acknowledge Alexander Zvinchuk, Louis Hamilton Data Management Center, Ukraine at the Institute of Pediatrics, Obstetrics and Gynecology in Kyiv for his work in preparing the UA-FCOU data and for providing instructional feedback about the cohort.

We are very grateful to all our colleagues working on the DRIVERS for Health Equity Consortium. In particular constructive reviews of this collaborative work were provided by the following: Linden Farrer, EuroHealthNet; Olle Lundberg, Stockholm University; and Diego Montano and Johannes Siegrist, University of Düsseldorf. Lastly, we would like to thank the peer reviewers who offered substantive feedback to earlier iterations of this manuscript.

All phases of this study were supported by a European Union's Seventh Framework Programme grant, 278350, as part of The Determinants to Reduce Health Inequity Via Early Childhood, Realising Fair Employment, and Social Protection (DRIVERS) research programme. The Czech ELSPAC Study (CZ-ELSPAC) was supported by the Ministry of Education of the Czech Republic (LM2011028, LO1214), and the Grant Agency of the Masaryk University (MUNI/M/1075/2013). The Northern Finland Birth Cohort (FI-NFBC8586) received financial support from the Academy of Finland; Biocenter, University of Oulu, Finland; the European Commission (EUROBLCS, Framework 5 Award QLG1-CT-2000-01643); EU FP7 EurHEALTHAgeing-277849; the Medical Research Council, UK (PrevMetSyn/SALVE); and the
MRC Centenary Early Career Award. The Amsterdam Born Children and their Development Study (NL$A B C D)$ received funding from the Netherlands Organization for Health Research and Development (ZonMw) Grant (TOP, 40-00812-98-11010). The All Babies in Southeast Sweden Study (SE-ABIS) has received financial support from the Juvenile Diabetes Research Foundation, the Swedish Child Diabetes Foundation, and the Research Council of Southeast Sweden.

Conflict of interest: The authors report no conflicts of interest.

\section{References}

1 Barker DJ, Gluckman PD, Godfrey KM, Harding JE, Owens J, Robinson J. Fetal nutrition and cardiovascular disease in adult life. Lancet 1993; 341:938-941.

2 Fuentes RM, Notkola IL, Shemeikka S, Tuomilehto J, Nissinen A. Tracking of body mass index during childhood: a 15-year prospective population-based family study in eastern Finland. International Journal of Obesity and Related Metabolic Disorders 2003; 27:716-721.

3 Johannsson E, Arngrimsson SA, Thorsdottir I, Sveinsson T. Tracking of overweight from early childhood to adolescence in cohorts born 1988 and 1994: overweight in a high birth weight population. International Journal of Obesity 2006; 30:1265-1271.

4 Cattaneo A, Monasta L, Stamatakis E, Lioret S, Castetbon K, Frenken F, et al. Overweight and obesity in infants and preschool children in the European Union: a review of existing data. Obesity Reviews 2010; 11:389-398.

5 Pillas D, Marmot M, Naicker K, Goldblatt P, Morrison J, Pikhart H. Social inequalities in early childhood health and development: a European-wide systematic review. Pediatric Research 2014; 76:418-424.

6 Siddiqi A, Irwin LG, Hertzman C. Total Environment Assessment Model for Early Child Development Vancouver: Human Early Learning Partnership (HELP), 2007.

7 Hawkins SS, Cole TJ, Law C. An ecological systems approach to examining risk factors for early childhood overweight: findings from the UK Millennium Cohort Study. Journal of Epidemiology and Community Health 2009; 63: 147-155.

8 Lobstein T, Jackson-Leach R, Moodie ML, Hall KD, Gortmaker SL, Swinburn BA, et al. Child and adolescent obesity: part of a bigger picture. Lancet 2015; 385:2510-2520.

9 Monasta L, Batty GD, Cattaneo A, Lutje V, Ronfani L, Van Lenthe FJ, et al. Early-life determinants of overweight and obesity: a review of systematic reviews. Obesity Reviews 2010; 11:695-708.

10 Suglia SF, Duarte CS, Chambers EC, Boynton-Jarrett R. Social and behavioral risk factors for obesity in early childhood. Journal of Developmental and Behavioral Pediatrics 2013; 34:549-556.

11 Robinson SM, Crozier SR, Harvey NC, Barton BD, Law CM, Godfrey KM, et al. Modifiable early-life risk factors for 
childhood adiposity and overweight: an analysis of their combined impact and potential for prevention. American Journal of Clinical Nutrition 2015; 101:368-375.

12 Dixon B, Pena MM, Taveras EM. Lifecourse approach to racial/ethnic disparities in childhood obesity. Advances in Nutrition 2012; 3:73-82.

13 Bammann K, Peplies J, De Henauw S, Hunsberger M, Molnar D, Moreno LA, et al. Early life course risk factors for childhood obesity: the IDEFICS case-control study. PLoS ONE 2014; 9:e86914.

14 Wisniewski AB, Chernausek SD. Gender in childhood obesity: family environment, hormones, and genes. Gender and Medicine 2009; 6(Suppl 1):76-85.

15 Ruiz M, Goldblatt P, Morrison J, Kukla L, Svancara J, Jarvelin MR, et al. Mother's education and the risk of preterm and small for gestational age birth: a DRIVERS meta-analysis of 12 European cohorts. Journal of Epidemiology and Community Health 2015; 69:826-833.

16 Veltsista A, Kanaka C, Gika A, Lekea V, Roma E, Bakoula C. Tracking of overweight and obesity in Greek youth. Obesity Facts 2010; 3:166-172.

17 Huus K, Ludvigsson JF, Enskar K, Ludvigsson J. Exclusive breastfeeding of Swedish children and its possible influence on the development of obesity: a prospective cohort study. BMC Pediatrics 2008; 8:1-6.

18 Cole TJ, Bellizzi MC, Flegal KM, Dietz WH. Establishing a standard definition for child overweight and obesity worldwide: international survey. BMJ 2000; 320:1240-1243.

19 Organisation for Economic Co-operation and Development. Classifying Educational Programmes: Manual for ISCED-97 Implementation in OECD Countries, 1999.

20 World Health Organisation Regional Office for Europe. European health for all database. http:// www.euro.who.int/en/data-and-evidence/databases/ european-health-for-all-database-hfa-db [last accessed January 2015].

21 Mackenbach JP, Kunst AE. Measuring the magnitude of socio-economic inequalities in health: an overview of available measures illustrated with two examples from Europe. Social Science \& Medicine 1997; 44:757-771.

22 Plewis I, Calderwood L, Hawkes D, Hughes G, Joshi H. The Millennium Cohort Study: Technical Report on Sampling, 4th edn. London: Bedford Group for Lifecourse and Statistical Studies, Institute of Education, University of London, 2007.

23 Pigeot I, Barba G, Chadjigeorgiou C, de Henauw S, Kourides $\mathrm{Y}$, Lissner $\mathrm{L}$, et al. Prevalence and determinants of childhood overweight and obesity in European countries: pooled analysis of the existing surveys within the IDEFICS Consortium. International Journal of Obesity 2009; 33:11031110.

24 Savva SC, Tornaritis M, Chadjigeorgiou C, Kourides YA, Savva ME, Panagi A, et al. Prevalence and sociodemographic associations of undernutrition and obesity among preschool children in Cyprus. European Journal of Clinical Nutrition 2005; 59:1259-1265.

25 Friedman LS, Lukyanova EM, Serdiuk A, Shkiryak-Nizhnyk ZA, Chislovska NV, Zvinchuk AV, et al. Social-environmental factors associated with elevated body mass index in a Ukrainian cohort of children. International Journal of Pediatric Obesity 2009; 4:81-90.

26 WHO Regional Office for Europe. The Challenge of Obesity in the WHO European Region and the Strategies for Response, 2007.

27 Closa-Monasterolo R, Ferre N, Luque V, Zaragoza-Jordana M, Grote $\mathrm{V}$, Weber $\mathrm{M}$, et al. Sex differences in the endocrine system in response to protein intake early in life. American Journal of Clinical Nutrition 2011; 94(6 Suppl):1920s-1927s.

28 Hornell A, Lagstrom H, Lande B, Thorsdottir I. Protein intake from 0 to 18 years of age and its relation to health: a systematic literature review for the 5th Nordic Nutrition Recommendations. Food and Nutrition Research 2013; 57:1-42.

29 Grontved A, Pedersen GS, Andersen LB, Kristensen PL, Moller NC, Froberg K. Personal characteristics and demographic factors associated with objectively measured physical activity in children attending preschool. Pediatric Exercise Science 2009; 21:209-219.

30 Brasholt M, Chawes B, Kreiner-Moller E, Vahlkvist S, Sinding M, Bisgaard H. Objective assessment of levels and patterns of physical activity in preschool children. Pediatric Research 2013; 74:333-338.

31 Lake A, Townshend T. Obesogenic environments: exploring the built and food environments. Journal of the Royal Society for the Promotion of Health 2006; 126:262-267.

32 Ulijaszek SJ. Frameworks of population obesity and the use of cultural consensus modeling in the study of environments contributing to obesity. Economics and Human Biology 2007; 5:443-457.

33 de Hoog ML, van Eijsden M, Stronks K, Gemke RJ, Vrijkotte TG. Overweight at age two years in a multi-ethnic cohort ( $\mathrm{BBCD}$ study): the role of prenatal factors, birth outcomes and postnatal factors. BMC Public Health 2011; 11:1-8.

34 European Commission. Europe 2020 Targets. http:/ / ec.europa.eu/europe2020/targets/eu-targets/index_en.htm [last accessed August 2015].

35 Kelishadi R, Azizi-Soleiman F. Controlling childhood obesity: a systematic review on strategies and challenges. Journal of Research in Medical Sciences 2014; 19:993-1008.

\section{Supporting Information}

Additional Supporting Information may be found in the online version of this article at the publisher's web-site:

Table S1. Prevalence of early childhood obesity by mother's educational level in 11 European birth cohorts, by gender.

Figure S1. Forest plot of combined (a) RII and (b) SII analyses of early childhood obesity risk in Europe, by gender. 